\title{
On bases in Banach spaces
}

\author{
by \\ TOMEK Bartoszyński (Arlington, VA), Mirna DžAmonja (Norwich), \\ Lorenz Halbeisen (Bern), Eva Murtinová (Praha) \\ and Anatolij Plichko (Kraków)
}

\begin{abstract}
We investigate various kinds of bases in infinite-dimensional Banach spaces. In particular, we consider the complexity of Hamel bases in separable and nonseparable Banach spaces and show that in a separable Banach space a Hamel basis cannot be analytic, whereas there are non-separable Hilbert spaces which have a discrete and closed Hamel basis. Further we investigate the existence of certain complete minimal systems in $\ell_{\infty}$ as well as in separable Banach spaces.
\end{abstract}

Outline. The paper is concerned with bases in infinite-dimensional Banach spaces. The first section contains the definitions of the various kinds of bases and biorthogonal systems and also summarizes some set-theoretic terminology and notation which will be used throughout the paper. The second section provides a survey of known or elementary results. The third section deals with Hamel bases and contains some consistency results proved using the forcing technique. The fourth section is devoted to complete minimal systems (including $\Phi$-bases and Auerbach bases) and the last section contains open problems.

1. Basics about bases. In what follows, all Banach spaces are assumed to be infinite-dimensional. Except one, all Banach spaces we consider are Banach spaces over the real field $\mathbb{R}$, and the only exception is the infinitedimensional Banach space $\mathbb{R}$ over the field $\mathbb{Q}$.

2000 Mathematics Subject Classification: Primary 46B20; Secondary 03E75, 03E35.

Key words and phrases: Hamel bases, complete minimal systems, $\Phi$-bases, Auerbach bases.

The research for this paper began during the "Workshop on Set Theory, Topology, and Banach Space Theory", which took place in June 2003 at Queen's University Belfast, whose hospitality is gratefully acknowledged. The workshop was supported by the Nuffield Foundation Grant NAL/00513/G of the third author, the EPSRC Advanced Fellowship of the second author and the grant GACR 201/03/0933 of the fourth author. 
Hamel bases. Let $X$ be a Banach space and let $\left\{x_{i}: i \in I\right\} \subseteq X$ be an arbitrary set of vectors of $X$. Let $\left\langle x_{i}: i \in I\right\rangle$ denote the linear span of $\left\{x_{i}: i \in I\right\}$. A set $\left\{x_{i}: i \in I\right\} \subseteq X$ is called a Hamel basis of $X$ if $\left\langle x_{i}: i \in I\right\rangle=X$ and for every $j \in I$ we have $x_{j} \notin\left\langle x_{i}: i \in I \backslash\{j\}\right\rangle$.

Hamel bases were first introduced by Georg Hamel in [Ham05] to define a discontinuous linear functional on the real line. In fact, he constructed by transfinite induction an algebraic basis in the Banach space $\mathbb{R}$ over $\mathbb{Q}$.

Complete minimal systems. Let $X$ be a Banach space and let $\left\{x_{i}: i \in I\right\}$ $\subseteq X$ be an arbitrary set of vectors of $X$. Let $\left[x_{i}: i \in I\right]$ denote the closure of the linear span of $\left\{x_{i}: i \in I\right\}$. A set $\left\{x_{i}: i \in I\right\} \subseteq X$ is called a complete system if $\left[x_{i}: i \in I\right]=X$, and it is called a minimal system if for every $j \in I, x_{j} \notin\left[x_{i}: i \in I \backslash\{j\}\right]$. A complete minimal system, abbreviated c.m.s., is a complete system which is also minimal.

Using functionals we can characterize minimal systems (and consequently complete minimal systems) in the following way (cf. [LT77, 1.f]):

Let $X$ be a Banach space. A pair of sequences $\left\{x_{i}: i \in I\right\} \subseteq X$ and $\left\{f_{i}: i \in I\right\} \subseteq X^{*}$ is called a biorthogonal system if $f_{j}\left(x_{i}\right)=\delta_{j}^{i}$. Now, a sequence $\left\{x_{i}: i \in I\right\} \subseteq X$ is minimal if and only if there is a sequence $\left\{f_{i}: i \in I\right\} \subseteq X^{*}$ such that the pair $\left(\left\{x_{i}: i \in I\right\},\left\{f_{i}: i \in I\right\}\right)$ is a biorthogonal system.

$\Phi$-bases. In [KPP88] Vladimir Kadets, Anatolij Plichko and Mikhail Popov introduced and investigated the notion of finitary bases of Banach spaces, called $\Phi$-bases, which are complete minimal systems of a certain type. $\Phi$-bases are weaker than the so-called Enflo-Rosenthal bases, which are complete minimal systems such that every countable subsystem is a basic sequence (i.e., a Schauder basis in the closure of its linear span) with respect to some enumeration of its elements.

If $\left\{x_{i}: 0 \leq i \leq n\right\} \subseteq X$ is any finite set of vectors of $X$, the basis constant $\mu\left\{x_{i}: 0 \leq i \leq n\right\}$ is the least number $M \leq \infty$ for which

$$
\left\|\sum_{i=0}^{k} a_{i} x_{i}\right\| \leq M \cdot\left\|\sum_{i=0}^{n} a_{i} x_{i}\right\|
$$

holds for any scalars $a_{i}$ and any integer $k$ with $0 \leq k \leq n$. A complete system $\left\{x_{i}: i \in I\right\} \subseteq X$ is called a finitary basis of $X$, briefly a $\Phi$-basis, if there exists a constant $M<\infty$ such that for any finite set $I_{0} \subseteq I$ there is an ordering $I_{0}=\left\{i_{j}: 0 \leq j \leq n\right\}$ such that $\mu\left\{x_{i_{j}}: 0 \leq j \leq n\right\} \leq M$. The least such constant $M$ is called the $\Phi$-basis constant of the $\Phi$-basis $\left\{x_{i}: i \in I\right\}$.

$\Phi$-bases are in fact just a special kind of complete minimal systems. To see this let us recall the following result (cf. [KPP88, Proposition 1]): 
Proposition 1.1. If $\left\{x_{i}: i \in I\right\} \subseteq X$ is a $\Phi$-basis of some Banach space $X$ with a $\Phi$-basis constant $M$, then the distance between any $x_{j} \in\left\{x_{i}: i \in I\right\}$ and $\left[x_{i}: i \in I \backslash\{j\}\right]$ is greater than or equal to $\frac{1}{2 M} \cdot\left\|x_{j}\right\|$.

Proof. By the definition of $M$ it is straightforward to see that for any $x_{j} \in\left\{x_{i}: i \in I_{0}\right\}$, where $I_{0} \subseteq I$ is a finite subset of $I$, for any set of scalars $a_{i}$ we have

$$
\left\|a_{j} x_{j}\right\| \leq 2 M \cdot\left\|\sum_{i \in I_{0}} a_{i} x_{i}\right\|
$$

and hence,

$$
2 M \cdot\left\|x_{j}-\sum_{i \in I_{0} \backslash\{j\}} a_{i} x_{i}\right\| \geq\left\|x_{j}\right\| .
$$

Thus, the distance between any $x_{j}$ and $\left[x_{i}: i \in I \backslash\{j\}\right]$ is greater than or equal to $\frac{1}{2 M} \cdot\left\|x_{j}\right\|$.

Now, assume that $\left\{x_{i}: i \in I\right\} \subseteq X$ is a normalized $\Phi$-basis of some Banach space $X$. By the previous fact and the Hahn-Banach Theorem, for every $i \in I$ we find an $f_{i} \in X^{*}$ such that $f_{i}\left(x_{j}\right)=\delta_{j}^{i}$, and moreover we can have $\left\|f_{i}\right\| \leq 2 M$ (for all $i \in I$ ). In particular $\left\{x_{i}: i \in I\right\} \subseteq X$ is a normalized complete minimal system.

Auerbach bases. In a finite-dimensional Hilbert space one may easily check that the vector $x$ is orthogonal to a vector $y$, denoted $x \perp y$, if and only if $\inf \{\|x-r y\|: r \in \mathbb{R}\}=\|x\|$. This can be used as a definition of orthogonality in any Banach space. In general this gives some surprising results, such as that the relation " $\perp$ " is not necessarily symmetric. Nevertheless one may still ask if every Banach space has a basis consisting of orthogonal vectors, more precisely an Auerbach basis as defined below.

Let $X$ be a Banach space and let $\left\{x_{i}: i \in I\right\} \subseteq X$. Then $\left\{x_{i}: i \in I\right\}$ is an Auerbach basis of $X$ if $\left[x_{i}: i \in I\right]=X$, and if for every $j \in I$,

$$
\left\|x_{j}\right\|=\inf \left\{\left\|x_{j}-y\right\|: y \in\left[x_{i}: i \in I \backslash\{j\}\right]\right\} .
$$

This notion was introduced by Herman Auerbach in his Ph.D. thesis [Au29] where he proved that every finite-dimensional normed space has an Auerbach basis, as mentioned in Stefan Banach's book [Ba32, p. 238]. The thesis and the proof were lost in World War II and Auerbach himself was killed by the Gestapo at Lwów in the summer of 1943. In 1947 Auerbach's theorem was reproved by Malon Day in [Da47] and Angus Taylor in [Ta47] and a very elegant proof can also be found in [LT77, p. 16].

Using biorthogonal systems we can characterize Auerbach bases as a special kind of complete minimal systems: 
Let $\left\{x_{i}: i \in I\right\}$ be a normalized c.m.s. of some Banach space $X$ and let $\left(\left\{x_{i}: i \in I\right\},\left\{f_{i}: i \in I\right\}\right)$ be the corresponding biorthogonal system. Then $\left\{x_{i}: i \in I\right\}$ is an Auerbach basis of $X$ if $\left\|f_{i}\right\|=1$ for every $i \in I$.

To "construct" a Hamel basis in some Banach space, we just well-order the vectors and then construct the Hamel basis by transfinite induction. So, every Banach space has a Hamel basis. However, the construction above uses the Axiom of Choice, and hence, we do not know how a Hamel basis looks like: For example, can a Hamel basis be closed, or non-meagre, or definable? We will answer some questions of that type in Section 3.

Unlike Hamel bases, not every Banach space has a c.m.s. (see, e.g., [Pl80] or [GK80]). Moreover, even though $\ell_{\infty}$ has a c.m.s. (see [DJ73] and [Go83]), the space $\ell_{\infty}$ has a non-separable subspace $X$ which has complete minimal systems, but none of them can be extended to a c.m.s. of $\ell_{\infty}$ (cf. [Go84, Theorem 3]). The existence of $\Phi$-bases and of Auerbach bases in certain Banach spaces will be discussed in Section 4.

Before going to the main part of the paper we need to review some basic set-theoretic notions.

Some set theory. For the reader's convenience we shall recall some settheoretic terminology and basic facts. Our set-theoretic axioms are the axioms of Zermelo and Fraenkel including the Axiom of Choice AC, denoted ZFC. All our set-theoretic notations and definitions are standard and can be found in textbooks such as [Je03], [Ku83] or [BJ95]. In some parts of this paper we use the so-called forcing technique to construct models of ZFC in which Banach spaces with certain properties exist. Forcing is a sophisticated tool and we do not attempt to explain it here. So, as far as forcing is concerned, the paper is not self-contained.

A set $x$ is transitive if every element of $x$ is a subset of $x$. A relation $R$ well-orders a set $x$, or $\langle R, x\rangle$ is a well-ordering, if $\langle R, x\rangle$ is a total ordering and every non-empty subset of $x$ has an $R$-least element. The Axiom of Choice is equivalent to the statement that every set can be well-ordered. A set $x$ is an ordinal number if $x$ is transitive and well-ordered by $\in$. Ordinal numbers will usually be denoted by Greek letters like $\alpha, \beta, \ldots$ In particular, for two ordinal numbers $\alpha$ and $\beta, \alpha<\beta$ is the same as saying $\alpha \in \beta$. The Axiom of Choice is also equivalent to the statement that for every set $x$ there exists an ordinal number $\alpha$ and a bijection $f: \alpha \rightarrow x$. The class of all ordinal numbers is transitive and well-ordered by $\in$. The set of all natural numbers is equal to the set of all finite ordinal numbers and is denoted by $\omega$. In particular, a natural number $n$ is the set of all natural numbers which are smaller than $n$, e.g., $0=\emptyset$. An ordinal number $\alpha$ is a called a successor ordinal if $\alpha=\beta \cup\{\beta\}$ (for some ordinal $\beta$ ), otherwise, $\alpha$ is called a limit ordinal. If $\alpha$ is an infinite limit ordinal, then the cofinality of $\alpha$, denoted 
$\operatorname{cf}(\alpha)$, is the least limit ordinal $\beta$ such that there is an increasing $\beta$-sequence $\left\langle\alpha_{\xi}: \xi<\beta\right\rangle$ with $\lim _{\xi \rightarrow \beta} \alpha_{\xi}=\alpha$ (see, e.g., [Je03, p. 31]).

For a set $x$ the cardinality of $x$, denoted by $|x|$, is the least ordinal number $\alpha$ for which there exists a bijection $f: \alpha \rightarrow x$; such an ordinal number $\alpha$ is called a cardinal number (or just a cardinal). For example, $|\omega|=\omega$, and finite cardinal numbers correspond to natural numbers. A set $x$ is called finite if $|x| \in \omega$, otherwise it is called infinite. Further, it is called countable if $|x| \leq \omega$. For a set $x$ the power set of $x$ is denoted by $\mathcal{P}(x)$. There exists a bijection between $\mathbb{R}$ and $\mathcal{P}(\omega)$, hence $|\mathbb{R}|=|\mathcal{P}(\omega)|$, and we denote this cardinality by $\mathfrak{c}$. The Continuum Hypothesis $\mathrm{CH}$ states that $\mathfrak{c}=\omega_{1}$, where $\omega_{1}$ denotes the least ordinal number which is not countable.

For any cardinals $\kappa$ and $\lambda, \kappa \cdot \lambda$ denotes the cardinality of the product $\kappa \times \lambda$. If at least one of the two cardinals is infinite, then $\kappa \cdot \lambda$ is always equal to $\max \{\kappa, \lambda\}$. For any cardinals $\kappa$ and $\lambda$ let $\kappa^{\lambda}$ denote the cardinality of the set ${ }^{\lambda} \kappa$ of all functions from $\lambda$ to $\kappa$. For example $2^{\lambda}=|\mathcal{P}(\lambda)|$ which is always strictly greater than $\lambda$. For any cardinal $\kappa$ let $\kappa^{+}$be the least cardinal which is strictly greater than $\kappa$. The Generalized Continuum Hypothesis GCH states that for each infinite cardinal $\kappa$ we have $2^{\kappa}=\kappa^{+}$. An infinite cardinal $\kappa$ is called regular if $\operatorname{cf}(\kappa)=\kappa$. Notice that $\operatorname{cf}(\kappa)$ is always regular. As a consequence of König's Theorem we get the following (see, e.g., [Je03, Corollaries 5.12-14]):

FACT 1.2. Let $\kappa$ and $\lambda$ be infinite cardinals. Then $\operatorname{cf}\left(2^{\kappa}\right)>\kappa, \operatorname{cf}\left(\kappa^{\lambda}\right)>\lambda$, and $\kappa^{\operatorname{cf}(\kappa)}>\kappa$.

For any set $x$ and any cardinal $\kappa$ let

$$
[x]^{\kappa}:=\{y \in \mathcal{P}(x):|y|=\kappa\} \quad \text { and } \quad[x]^{<\kappa}:=\{y \in \mathcal{P}(x):|y|<\kappa\} .
$$

If $x$ is infinite, then $\left|[x]^{<\omega}\right|=|x|$.

2. Cardinality issues in Banach spaces. In [HH00] (see also [Ma45]) it is shown that for any infinite-dimensional Banach space $X$, and for any Hamel basis $H$ of $X$ we have $|H|=|X|$, which is at least $\mathfrak{c}$. (Note that the point of this result is when $|X|=\mathfrak{c}$.) This implies the following

Proposition 2.1. Every Banach space $X$ over a complete field has $2^{|X|}$ different normalized Hamel bases.

Proof. Let $H \subseteq X$ be a normalized Hamel basis of $X$ and let $h_{0} \in H$. For any set $I \subseteq H \backslash\left\{h_{0}\right\}$, let $B_{I}:=\left\{\left(h_{0}+h\right) /\left\|h_{0}+h\right\|: h \in I\right\}$ and let $H_{I}:=B_{I} \cup(H \backslash I)$. Now, $H_{I}$ is a normalized Hamel basis of $X$ and for any two different subsets $I$ and $I^{\prime}$ of $H \backslash\left\{h_{0}\right\}$ we have $H_{I} \neq H_{I^{\prime}}$. Since there are ${ }_{2}{ }^{|X|}$ such subsets, $X$ has $2^{|X|}$ different normalized Hamel bases.

Can we ask for more? Obviously, one cannot aim for more than $2^{\kappa}$ different normalized Hamel bases, but one could try to find a family of $2^{\kappa}$ different 
normalized Hamel bases such that the cardinality of the intersection of any two of them is less than $\kappa$ (see Question 4 in Section 5).

Proposition 2.2. The unit sphere of a real Banach space $X$ is not the union of fewer than $\mathfrak{c}$ Hamel bases of $X$.

Proof. Let $x$ and $y$ be two different unit vectors of $X$ and define $S=$ $\{(r x+t y) /\|r x+t y\|: r, t \in \mathbb{R}\}$. Then $S$ is a subset of the unit sphere with $|S|=\mathfrak{c}$ and every Hamel basis of $X$ contains at most two vectors from $S$. Thus $S$, and in particular the unit sphere, cannot be covered by fewer than c Hamel bases of $X$.

At this point we would like to mention that not even a weakened form of Proposition 2.2 works for the Banach space $\mathbb{R}$ over $\mathbb{Q}$ : In fact Paul Erdôs and Shizuo Kakutani showed in [EK43, Theorem 2] that $\mathrm{CH}$ is equivalent to the statement that $\mathbb{R}$ is the union of countably many sets of rationally independent numbers.

With respect to complete minimal systems we get the following

Proposition 2.3. The cardinality of a c.m.s. of a Banach space $X$ is equal to the density character of $X$ (denoted by $\mathrm{d}(X))$.

Proof. On the one hand, the set of all finite linear combinations of a c.m.s. with rational coefficients is dense in $X$, and on the other hand, every c.m.s. of $X$ is discrete in $X$.

At this point we would like to introduce the notation $B_{x, r}$ for the open ball centred at $x$ with radius $r$, which will be useful throughout the paper.

As a matter of fact we would like to mention the following simple observations, as we shall use them later:

Proposition 2.4. Let $X$ be a Banach space.

(a) If $A \subseteq X$ and $|A|<\mathrm{d}(X)$, then $A$ is nowhere dense in $X$.

(b) We always have $|X| \leq \mathrm{d}(X)^{\omega}$ (see also Lemma 2.8).

Proof. (a) Suppose otherwise, so let $B_{x, r}$ be an open ball in which $A$ is dense. (Clearly this implies that $A$ is infinite.) Then $\bigcup_{q \in \mathbb{Q}} q(A-x)$ is a set of the same size as $A$ and is dense in $X$.

(b) If $D$ is a dense subset of $X$ then every element of $X$ is a limit point of a countable sequence from $D$.

The following is a well known fact about metric spaces.

FACT 2.5. For every infinite- or finite-dimensional Banach space $X$ we have $\mathrm{d}(X)=\mathrm{w}(X)$ (where $\mathrm{w}(X)$ denotes the weight of the space $X)$.

Corollary 2.6. The number of open (and hence of closed) subsets of a Banach space $X$ is at most $2^{\mathrm{d}(X)}$. In particular, $|X| \leq 2^{\mathrm{d}(X)}$ (which also follows from Lemma 2.8 below). 
Proof. Every open set is the union of some family of basic open sets and every point in a Banach space is a closed set.

Using these facts we can prove the following:

Theorem 2.7. For any Banach space $X$ we have $\operatorname{cf}(|X|)>\omega$.

In order to prove this theorem we need the following

Lemma 2.8 (Juhász-Szentmiklóssy). For any Banach space $X$ we have $\mathrm{d}(X)^{\omega} \leq|X|$. Consequently, by Proposition 2.4(b), $|X|=\mathrm{d}(X)^{\omega}$.

Proof. Let $X$ be an infinite-dimensional Banach space with $\mathrm{d}(X)=\lambda$, which, by Fact 2.5 , is the same as $\mathrm{w}(X)$. First note that by the Bing Metrization Theorem (cf. [Bi51]), every metric space of weight $\lambda$ contains $\lambda$ pairwise disjoint open sets. Consequently, since every open subset of $X$ has the same weight as $X$ itself, every open subset of $X$ contains $\lambda$ pairwise disjoint open sets. Now start with $\lambda$ pairwise disjoint open balls, inside of each take $\lambda$ pairwise disjoint open balls and so on. The tree we get in this way is a tree of height $\omega$ which contains $\lambda^{\omega}$ different branches, and since the diameters of the open sets converge to 0 , every branch yields a Cauchy sequence. Hence, by the completeness of $X$ we have $\lambda^{\omega} \leq|X|$.

Now we are ready to prove the theorem.

Proof of Theorem 2.7. Let $X$ be an infinite-dimensional Banach space of cardinality $\kappa$ with $\mathrm{d}(X)=\lambda$. By Lemma 2.8 and by Proposition 2.4(b), $\lambda^{\omega}=\kappa$, and hence, by Fact $1.2, \operatorname{cf}(\kappa)>\omega$.

\section{The complexity of Hamel bases}

3.1. The general case. Many arguments about Banach spaces involve the Baire Category Theorem the content of which we recall briefly. Let $X$ be a Banach space. Since $X$ is a complete metric space, $X$ is a so-called Baire space, i.e., a space in which non-empty open sets are non-meagre. Equivalently, each intersection of countably many open dense sets in $X$ is dense in $X$. A subset $A$ of $X$ has the Baire property if there is an open set $\mathcal{O}$ such that $\mathcal{O} \triangle A$ is meagre (i.e., of first category), where $\mathcal{O} \triangle A=$ $(\mathcal{O} \backslash A) \cup(A \backslash \mathcal{O})$.

The ideal of meagre sets in a space $X$ will be denoted by $\mathcal{M}_{X}$. Its cofinality $\operatorname{cof}\left(\mathcal{M}_{X}\right)$ is the smallest size of a subfamily $\mathcal{F}$ of $\mathcal{M}_{X}$ such that every meagre set is contained in an element of $\mathcal{F}$. Noticing that $F_{\sigma}$ meagre sets are cofinal in $\mathcal{M}_{X}$ we may redefine $\operatorname{cof}\left(\mathcal{M}_{X}\right)$ as the smallest size of a subfamily of $F_{\sigma}$ meagre sets that is cofinal for the $F_{\sigma}$ meagre sets.

Let us first prove the following two results:

Proposition 3.1. Suppose that $X$ is any Banach space and that $H$ is a Hamel basis of $X$. If $H$ has the Baire property, then $H$ is meagre. 
Proof. Let $H$ be a Hamel basis of $X$ and assume that it has the Baire property but is non-meagre. Then there is a non-empty open set $\mathcal{O}$ such that $\mathcal{O} \triangle H$ is meagre. Let $h \in H \cap \mathcal{O}$ and let $x_{i}(i<\omega)$ be a sequence of vectors converging to $h$ such that each $x_{i}$ needs at least four vectors from $H$ to represent it in the basis $H$. Such a sequence exists, since we can just take any converging sequence and then add some small linear combinations of $H$ to it. Now, since the $x_{i}$ 's converge to $h$ and $\mathcal{O}$ is open, there is some $j<\omega$ such that $(h+\mathcal{O}) \cap\left(x_{j}+\mathcal{O}\right) \neq \emptyset$, in particular it is open. Further, since $h \in H \cap \mathcal{O}$, we have $(h+H) \cap\left(x_{j}+H\right) \neq \emptyset$, and by the property of $x_{j}$, this contradicts the fact that $H$ is a Hamel basis.

Proposition 3.2. Every Banach space over a complete field contains a Hamel basis which is nowhere dense and one which is dense and meagre.

Proof. Let $X$ be a Banach space over some complete field, and let $\left\{B_{\alpha}\right.$ : $\alpha<\lambda\}$ be its open base, where $\lambda$ is the weight of $X$.

By transfinite induction we can construct a linearly independent set $H^{\prime}=$ $\left\{h_{\alpha}: \alpha<\lambda\right\}$ in $X$ such that for every $\alpha<\lambda$ we have $h_{\alpha} \in B_{\alpha}$ and $\left\|h_{\alpha}\right\| \in \mathbb{Q}$. Why? Assume we have already constructed a linearly independent set $H_{\beta}=\left\{h_{\alpha}: \alpha<\beta\right\}$ for some $\beta<\lambda$. Let $\left\langle H_{\beta}\right\rangle$ denote the linear span of $H_{\beta}$. Since $\beta<\lambda$, we have $B_{\beta} \nsubseteq\left\langle H_{\beta}\right\rangle$, and therefore we can find a vector $h \in B_{\beta} \backslash\left\langle H_{\beta}\right\rangle$. Pick $q \in(\|h\|-\varepsilon,\|h\|+\varepsilon) \cap \mathbb{Q}$, where $\varepsilon>0$ is such that $B_{h, \varepsilon} \subset B_{\beta}$. Let $h_{\beta}=q \cdot h /\|h\| ;$ then $h_{\beta} \in B_{\beta}$ and $\left\|h_{\beta}\right\| \in \mathbb{Q}$.

Now extend $H^{\prime}$ by unit vectors to a Hamel basis $H$ of $X$. By construction, $H$ is a Hamel basis of $X$ which is dense in $X$. Moreover, for every positive rational $q$ the set $\{h \in H:\|h\|=q\}$ is nowhere dense because it is contained in a sphere. This implies that $H$, as the union of countably many nowhere dense sets, is meagre.

Define $H^{\sim}=\{h /\|h\|: h \in H\}$. Then $H^{\sim}$ is a Hamel basis of $X$ which is nowhere dense.

By transfinite induction one can show that every separable Banach space contains a Hamel basis which is non-meagre (see [GMP83]). In fact, we can prove a slightly more general result:

Theorem 3.3. Let $X$ be a Banach space satisfying $\operatorname{cof}\left(\mathcal{M}_{X}\right) \leq|X|$. Then $X$ has a non-meagre Hamel basis.

Proof. Let $X$ be a Banach space satisfying the assumptions and let $\left\{B_{\alpha}\right.$ : $\alpha<\kappa\}$ be an enumeration of a cofinal family of meagre $F_{\sigma}$ sets of the least possible cardinality. Hence $|X| \geq \kappa$ by the assumptions. First we construct by induction on $\alpha$ a non-meagre set $H^{\prime}=\left\{h_{\alpha}: \alpha<\kappa\right\}$ of linearly independent vectors. Assume we have already chosen the set $H_{\alpha}^{\prime}=\left\{h_{\beta}: \beta<\alpha\right\}$ for some $\alpha<\kappa$. Now, there is an $h_{\alpha}$ such that $h_{\alpha} \notin\left\langle H_{\alpha}^{\prime}\right\rangle \cup B_{\alpha}$. Why? Since $|X| \geq \kappa$ the set $H_{\alpha}^{\prime}$ cannot be a Hamel basis of $X$, and therefore $\left\langle H_{\alpha}^{\prime}\right\rangle$ is a proper 
subset of $X$. We choose a (non-zero) $x^{\prime} \in X \backslash\left\langle H_{\alpha}^{\prime}\right\rangle$. If $\left\langle H_{\alpha}^{\prime}\right\rangle \cup B_{\alpha}=X$, then the set $A=X \backslash B_{\alpha}$ is contained in $\left\langle H_{\alpha}^{\prime}\right\rangle$ and is hence disjoint from $x^{\prime}+\left\langle H_{\alpha}^{\prime}\right\rangle$ and in particular from $x^{\prime}+A$. However, since $B_{\alpha}$ is meagre $F_{\sigma}$, both $A$ and $x^{\prime}+A$ are countable intersections of open dense sets and hence the Baire Category Theorem implies that the intersection of $A$ and $x^{\prime}+A$ must be dense, a contradiction. Hence, $\left\langle H_{\alpha}^{\prime}\right\rangle \cup B_{\alpha} \neq X$ and we can choose a (non-zero) $h_{\alpha} \in X \backslash\left(\left\langle H_{\alpha}^{\prime}\right\rangle \cup B_{\alpha}\right)$.

Finally, let $H^{\prime}=\bigcup_{\alpha<\kappa} H_{\alpha}^{\prime}$ and let $H$ be a Hamel basis of $X$ containing $H^{\prime}$. Then, by construction, the set $H$ is not contained in any meagre set and therefore cannot be meagre.

Corollary 3.4. If $X$ is a Banach space satisfying $2^{\mathrm{d}(X)} \leq|X|$, then $X$ contains a non-meagre Hamel basis. In particular, every separable Banach space has a non-meagre Hamel basis.

Proof. Since every nowhere dense set is contained in some closed set whose complement is open dense, and since $\mathrm{d}(X)=\mathrm{w}(X)$, there are at most $2^{\mathrm{d}(X)}$ different open dense sets in $X$. This implies that $\operatorname{cof}\left(\mathcal{M}_{X}\right) \leq$ $\left(2^{\mathrm{d}(X)}\right)^{\omega}=2^{\mathrm{d}(X) \cdot \omega}=2^{\mathrm{d}(X)}$, hence, by Theorem $3.3, X$ contains a non-meagre Hamel basis. In particular, for separable spaces $X$ we have $\mathrm{d}(X)=\omega$, which implies $\operatorname{cof}\left(\mathcal{M}_{X}\right) \leq 2^{\mathrm{d}(X)}=\mathfrak{c} \leq|X|$, and therefore, every separable Banach space has a non-meagre Hamel basis.

The problem whether every Banach space contains a non-meagre Hamel basis will be discussed again in Section 3.4.

The following theorem was proved in [Hal01] and we shall use it on several occasions. Before we state the theorem let us recall that a subset $S$ of a Banach space $X$ is called linearly Baire if for every positive integer $n$ the set of all linear combinations involving exactly $n$ vectors of $S$ has the Baire property.

THEOREM 3.5. If $X$ is a Banach space over any field $\mathbb{F}$ and $H$ is a Hamel basis of $X$, then $H$ is not linearly Baire.

To keep the notation short, let us introduce the following definition. Let $X$ be a Banach space over the field $\mathbb{F}$ and let $H \subseteq X$. For a positive integer $n$, let $[H]^{n}$ be the set of all $n$-element subsets of $H$ and let

$$
H_{n}:=\left\{\sum_{i=1}^{n} \alpha_{i} h_{i}: \alpha_{1}, \ldots, \alpha_{n} \in \mathbb{F} \backslash\{0\} \text { and }\left\{h_{1}, \ldots, h_{n}\right\} \in[H]^{n}\right\} .
$$

A reformulation of Theorem 3.5 that we shall use below is

Corollary 3.6. Let $X$ be a Banach space over the field $\mathbb{F}$ and let $\Gamma$ be a family of subsets of $X$ such that every set in $\Gamma$ has the property of Baire and for every natural number $n$ and $H \in \Gamma$, the set $H_{n}$ is in $\Gamma$. Then no set in $\Gamma$ is a Hamel basis for $X$. 
Another consequence of this result is

Theorem 3.7. No Banach space $X$ has a Hamel basis that is $\sigma$-compact.

Proof. Let $X$ be a Banach space. To better illustrate the method of the proof let us first show that $X$ cannot have a compact Hamel basis. So suppose towards a contradiction that $H$ were such. Hence for every $a \leq b$ in $\mathbb{R}$ the set $[a, b] \cdot H$ is compact and so is any finite sum of such sets since for any compact $K$ the set $K+K$ is compact. In this way we deduce that $H_{1}=\bigcup_{n=1}^{\infty}[-n, n] \cdot H \backslash\{0\}, H_{1} \cup H_{2}=\bigcup_{n=1}^{\infty}\{[-n, n] \cdot H+[-n, n] \cdot H\} \backslash\{0\}$ etc. are all Borel and so $H$ is linearly Baire, in contradiction with Theorem 3.5.

The proof for $\sigma$-compactness is the same, noticing that if $H=\bigcup_{n<\omega} K_{n}$ then for example $H+H=\bigcup_{n<\omega, m<\omega}\left(K_{n}+K_{m}\right)$, and the other sets involved in checking that $H$ is linearly Baire have similar definitions.

As opposed to compact sets, closed sets $C$ do not necessarily satisfy that $C+C$ is closed and in fact in Section 3.2 we shall see an example of a Banach space that has a closed Hamel basis. This space is non-separable and by Theorem 3.10 this assumption is necessary.

3.2. The non-separable case

THEOREM 3.8. There are non-separable Banach spaces which have a closed Hamel basis. Moreover, there are Hilbert spaces of arbitrarily large cardinality which have a discrete and closed Hamel basis.

Proof. Let $\kappa$ be an arbitrarily large cardinal satisfying $\kappa^{\omega}=\kappa$ (for example for any $\lambda$ we may let $\left.\kappa=\lambda^{\omega}\right)$. Further, let $\ell_{2}(\kappa)$ be the Hilbert space of all functions $f: \kappa \rightarrow \mathbb{R}$ with

$$
\|f\|:=\sqrt{\sum_{\beta<\kappa} f(\beta)^{2}}<\infty .
$$

Notice that every $f \in \ell_{2}(\kappa)$ must have countable (or finite) support, i.e., the set $\{\beta<\kappa: f(\beta) \neq 0\}$ is at most countable.

We shall see that $\ell_{2}(\kappa)$ has a discrete and closed Hamel basis. Note that $\left|\ell_{2}(\kappa)\right|=\kappa$ since $\kappa^{\omega}=\kappa$.

Let $X$ be the direct sum of $\omega_{1}$ copies of $\ell_{2}(\kappa)$ with the $\ell_{2}$-norm. By the definition of $\kappa$ it is easy to see that $\left|\ell_{2}(\kappa)\right|=|X|=\kappa$ and that $X$ and $\ell_{2}(\kappa)$ are essentially the same space, so $X$ is a Hilbert space of cardinality $\kappa$. For $\alpha<\omega_{1}$, let $Y_{\alpha}$ be the $\alpha$ 's copy of $\ell_{2}(\kappa)$ (with respect to the direct sum $X$ ) and let $E_{\alpha}=\left\{e_{\iota}^{\alpha}: \iota<\kappa\right\}$ be the canonical orthonormal vectors of $Y_{\alpha}$, i.e., $e_{\iota}^{\alpha}(\beta)=\delta_{\beta}^{\iota}$ (for all $\left.\beta<\kappa\right)$. Further, for $\alpha<\omega_{1}$ let

$$
X_{\alpha}=\bigoplus_{\eta \leq \alpha} Y_{\eta},
$$

so $X=\bigcup_{\alpha<\omega_{1}} X_{\alpha}$. 
Let $H_{0}=\left\{x_{\iota}^{0}: \iota<\kappa\right\}$ be a Hamel basis for $X_{0}$ and let

$$
B_{1}=\left\{x_{\iota}^{0}+e_{\iota}^{1}: \iota<\kappa\right\} \cup\left\{e_{\iota}^{1}: \iota<\kappa\right\} .
$$

Then $B_{1}$ is a linearly independent set of vectors which is closed in $X_{1}$ - since it does not contain any converging sequence - and whose linear span contains $Y_{0} \subseteq X_{1}$, as $x_{\iota}^{0}=x_{\iota}^{0}+e_{\iota}^{1}-e_{\iota}^{1}$. However, $B_{1}$ is not a Hamel basis for $X_{1}$. Let $H_{1}$ be a Hamel basis of $Y_{1}$ extending $E_{1}$ and let $\left\{x_{\iota}^{1}: \iota<\kappa\right\}=H_{1} \backslash E_{1}$.

We proceed now by transfinite induction. For successor ordinals $\alpha+1$ $<\omega_{1}$ we define

- $B_{\alpha+1}:=B_{\alpha} \cup\left\{x_{\iota}^{\alpha}+e_{\iota}^{\alpha+1}: \iota<\kappa\right\} \cup\left\{e_{\iota}^{\alpha+1}: \iota<\kappa\right\}$,

- $H_{\alpha+1}$ is a Hamel basis of $Y_{\alpha+1}$ extending $E_{\alpha+1}$, and

- $\left\{x_{\iota}^{\alpha+1}: \iota<\kappa\right\}=H_{\alpha+1} \backslash E_{\alpha+1}$.

By induction, $B_{\alpha+1}$ is a set of linearly independent vectors whose linear span contains $X_{\alpha}$. Further, for limit ordinals $\gamma<\omega_{1}$ we define

- $B_{\gamma}=\bigcup_{\alpha<\gamma} B_{\alpha}$

- $H_{\gamma}$ is a Hamel basis of $X_{\gamma}$ extending $B_{\gamma}$, and

- $\left\{x_{\iota}^{\gamma}: \iota<\kappa\right\}=H_{\gamma} \backslash B_{\gamma}$.

For $\alpha<\beta \leq \gamma$ we have $B_{\alpha} \subseteq B_{\beta} \subseteq B_{\gamma}$, and since, by induction, $B_{\alpha}$ and $B_{\beta}$ are sets of linearly independent vectors, also $B_{\gamma}$ is a set of linearly independent vectors. Further note that $H_{\gamma} \backslash B_{\gamma}$ is non-empty. Moreover, $\left|H_{\gamma} \backslash B_{\gamma}\right|=\kappa$ because taking a cofinal sequence $\left\langle\gamma_{n}: n<\omega\right\rangle$ in $\gamma$, we see that no vector of the form $\sum_{n<\omega} 2^{-n} x_{\iota(n)}^{\gamma_{n}}$ where $\iota(n)<\kappa$ is in $\left\langle B_{\gamma}\right\rangle$. Finally, let

$$
H=\bigcup_{\alpha<\omega_{1}} B_{\alpha} ;
$$

then, by construction, $H$ is discrete and closed in $X$, and since every vector in $X$ has countable support, $H$ is a Hamel basis of the Hilbert space $X$.

3.3. The separable case. It may be conjectured from Corollary 3.4 that separable Banach spaces behave with respect to the Hamel bases similarly to the space $\mathbb{R}$ considered as a vector space over $\mathbb{Q}$. We shall give some further remarks which seem to support this statement. Let us first show that a Hamel basis in a separable Banach space over $\mathbb{R}$ cannot be a Borel or an analytic set. In order to do so, we have to first recall a basic property of $\boldsymbol{\Sigma}_{n}^{1}$ sets (see also [Ke95, Chapter V]):

For each $n \geq 1$ we define the projective classes $\boldsymbol{\Sigma}_{n}^{1}$ and $\boldsymbol{\Pi}_{n}^{1}$ of sets in a Polish space $X$ as follows: $\boldsymbol{\Sigma}_{1}^{1}$ is the collection of all analytic sets (i.e., projections of closed sets in $\left.X \times{ }^{\omega} \omega\right)$ and $\Pi_{1}^{1}$ is the collection of the complements of analytic sets. Further, $\boldsymbol{\Sigma}_{n+1}^{1}$ is the collection of projections of $\boldsymbol{\Pi}_{n}^{1}$ sets in $X \times{ }^{\omega} \omega$, and $\boldsymbol{\Pi}_{n+1}^{1}$ is the collection of the complements of $\boldsymbol{\Sigma}_{n+1}^{1}$ sets. 
Finally, a subset $S$ of $X$ is called a projective set of $X$ if there is a positive integer $n$ such that $S$ belongs to $\boldsymbol{\Sigma}_{n}^{1}$.

Now, the classes $\Sigma_{n}^{1}$ are closed under images and preimages of continuous functions between Polish spaces (cf. [Ke95, Proposition 37.1]).

Lemma 3.9. Suppose that $X$ is a separable Banach space. Then for every $\boldsymbol{\Sigma}_{n}^{1}$ set $H$ and every positive integer $m, \bigcup_{i \leq m} H_{i}$ is a $\boldsymbol{\Sigma}_{n}^{1}$ set.

Proof. Let $H \subseteq X$ be a $\boldsymbol{\Sigma}_{n}^{1}$ set in $X$. As $\mathbb{R}$ is a Polish space, $H \times(\mathbb{R} \backslash\{0\})$ is a $\boldsymbol{\Sigma}_{n}^{1}$ set in $X \times \mathbb{R}$. Define the function $f: X \times \mathbb{R} \rightarrow X$ by stipulating $f(x, r):=r x$. Then $f$ is continuous and by the previous facts we deduce that $f[H \times(\mathbb{R} \backslash\{0\})]=\{r h: r \in \mathbb{R} \backslash\{0\}, h \in H\}$ is a $\boldsymbol{\Sigma}_{n}^{1}$ set in $X$, which shows that $H_{1}$ is a $\boldsymbol{\Sigma}_{n}^{1}$ set in $X$. Further, if $H^{\prime}$ and $H^{\prime \prime}$ are both $\boldsymbol{\Sigma}_{n}^{1}$ sets, then $H^{\prime}+H^{\prime \prime}$, as the image of the continuous function $X \times X \rightarrow X+X$, is again a $\boldsymbol{\Sigma}_{n}^{1}$ set.

Since all analytic sets have the Baire property, by Lemma 3.9 and the proof of Proposition 3.1 we get the following: If an analytic set $H$ is a Hamel basis of a separable Banach space, and if $H_{1}$ has the Baire property, then $H_{1}$ is meagre. However, the next result shows that a Hamel basis of such a Banach space can never be an analytic set.

THEOREM 3.10. If $X$ is a separable Banach space and $H$ is a Hamel basis of $X$, then $H$ is not an analytic set.

Proof. Suppose $H \subseteq X$ is an analytic Hamel basis of $X$. By Lemma 3.9, for every natural number $n$ the set $\bigcup_{i<n} H_{i}$ is analytic. Now, by Theorem 3.5 it follows that there is an $n_{0}$ for which $H_{n_{0}}$ does not have the Baire property. But $H_{n_{0}}=\bigcup_{i<n_{0}} H_{i} \backslash \bigcup_{i<n_{0}} H_{i}$, and therefore, as the difference of two sets having the Baire property, $H_{n_{0}}$ must have the Baire property as well-a contradiction.

It is (relatively) consistent with ZFC that all projective sets in $\mathbb{R}$ have the property of Baire, by a theorem of Saharon Shelah in [Sh84]. We shall use this in Section 3.5 to see that it is consistent that no separable Banach space over $\mathbb{R}$ has a Hamel basis that is a projective set.

3.4. Consistency results. In modern set theory, one usually gets consistency results by a forcing construction. Forcing was invented by Paul Cohen in the early 1960s to show that AC as well as CH are not provable in ZermeloFraenkel Set Theory ZF. In fact he showed that $\neg A C$ is relatively consistent with ZF and that $\neg \mathrm{CH}$ is relatively consistent with ZFC. (Apart from this paragraph, we use the common set-theoretic shorthand where "consistent" stands for "relatively consistent"). Forcing is a technique to extend models of set theory in such a way that certain statements become true in the extension, no matter if they were true or false in the ground model. In other 
words, forcing adds new sets to some ground model and by choosing the right forcing notion we can make sure that the new sets have some desired properties. For a short introduction to forcing we refer the reader to [Je86]. To get consistency results with respect to Hamel bases we first have to define a notion of forcing, i.e., a partial order, which adds new Hamel bases with certain properties to the ground model. So, let us first introduce a forcing notion which does the job:

In the following, let $X$ be an arbitrary but fixed real Banach space of cardinality $\kappa$ and let $\lambda$ be a cardinal less than or equal to $\kappa$. With respect to the space $X$, let $\mathbb{B}_{\lambda}=\left\langle B_{\lambda}, \leq\right\rangle$ be the following partially ordered set. A so-called condition $p \in B_{\lambda}$ consists of less than $\lambda$ linearly independent vectors of $X$ and for $p, q \in B_{\lambda}$ let $p \leq q$ if and only if $p \subseteq q$.

Our goal is to show that, for $\lambda=\operatorname{cf}(\kappa)>\omega$, forcing with $\mathbb{B}_{\lambda}$ adds a Hamel basis of $X$ which is non-meagre. For this we have to make sure that the Banach space $X$ in the extension is very much the same as in the ground model, i.e., we would not like to add new vectors to $X$, but how can we do this? We may consider a Banach space $X$ as a set of vectors belonging to some universe $\mathbf{V}$. This set is denoted by $X^{\mathbf{V}}$. Now, if we extend $\mathbf{V}$, then the set $X^{\mathbf{V}}$ still exists in the extension but may have some other properties than in the ground model $\mathbf{V}$. For example the norm on $X^{\mathbf{V}}$ in the extension might no longer be complete or the definition of $X^{\mathbf{V}}$ might have changed in the extension. However, in most of the cases the "definition" or "construction" of the Banach space $X$ is the same in $\mathbf{V}$ as in the extension, so, forcing with $\mathbb{B}_{\lambda}$ does not change the space in some sense and we will call such spaces conservative.

Before we can give some examples of conservative spaces we have to know more about the forcing notion $\mathbb{B}_{\lambda}$.

Let $\lambda$ be an infinite cardinal. A forcing notion $\mathbb{P}=\langle P, \leq\rangle$ is called $\lambda$ closed if for any increasing sequence $p_{0} \leq \cdots \leq p_{\alpha} \leq \cdots$ in $P$ of length $\gamma<\lambda$ there is a $q \in P$ such that $q \geq p_{\alpha}$ for all $\alpha<\gamma$. A forcing notion $\mathbb{P}$ which is $\lambda$-closed does not add new bounded subsets to $\lambda$ and does not collapse any cardinals less than or equal to $\lambda$. In particular, if $\lambda>\omega$ then a $\lambda$-closed forcing notion does not add new reals.

Let us turn back to the forcing notion $\mathbb{B}_{\lambda}$ : Let $X$ be a Banach space and let $\lambda=\operatorname{cf}(\kappa)$, where $\kappa=|X|$. Then $\lambda$ is a regular uncountable cardinal, which implies that any increasing sequence $p_{0} \leq \cdots \leq p_{\alpha} \leq \cdots$ of conditions of $\mathbb{B}_{\lambda}$ of length less than $\lambda$ has an upper bound, and thus $\mathbb{B}_{\lambda}$ is $\lambda$-closed. This tells us that forcing with $\mathbb{B}_{\lambda}$ does not collapse any cardinals less than or equal to $\lambda$. Moreover, since $\lambda>\omega$, forcing with $\mathbb{B}_{\lambda}$ does not add any new reals.

Let us now give some examples of conservative spaces: For $1 \leq p \leq \infty$, all $\ell_{p}$ and $L_{p}$ spaces, as well as all $\ell_{p}(\mathfrak{c})$ spaces (and for regular $\lambda$ even all 
$\ell_{p}(\lambda)$ spaces) are conservative. All these spaces are present in every universe of ZFC. As an illustration let us demonstrate that $\ell_{\infty}(\mathfrak{c})$ is conservative: First notice that every vector $x$ in $\ell_{\infty}(\mathfrak{c})$ is a sequence of real numbers of length $\mathfrak{c}$, so $x$ is an element of $\mathfrak{c} \mathbb{R}$, which implies $\kappa=\left|\ell_{\infty}(\mathfrak{c})\right|=2^{\mathfrak{c}}>\mathfrak{c}$. By Fact 1.2 we get $\operatorname{cf}(\kappa)=\operatorname{cf}\left(2^{\mathfrak{c}}\right)>\mathfrak{c}>\omega$. Now, since $x \in{ }^{\mathfrak{c}} \mathbb{R}={ }^{\mathfrak{c}} \mathfrak{c}$, it can be encoded as a subset of $\mathfrak{c} \times \mathfrak{c}$ of cardinality $\mathfrak{c}$, and since $|\mathfrak{c} \times \mathfrak{c}|=\mathfrak{c}$, every $x \in \ell_{\infty}(\mathfrak{c})$ can be encoded as a subset of $\mathfrak{c}$ (which is a subset of $\kappa$ ) of cardinality $\mathfrak{c}$, where $\mathfrak{c}$ is strictly less than $\mathrm{cf}(\kappa)$. Since this encoding is done in an absolute way (i.e., not depending on the underlying universe of ZFC) the space will not change unless we add bounded subsets to $\operatorname{cf}(\kappa)$.

Now let us prove the following

THEOREM 3.11. Let $X$ be a Banach space in some universe $\mathbf{V}$ of ZFC in which $X$ has cardinality $\kappa$ and in which $\theta<\operatorname{cf}(\kappa)$ implies $\theta^{\omega}<\kappa$. Then there exists a $\mathrm{cf}(\kappa)$-closed forcing extension of $\mathbf{V}$ in which $X^{\mathbf{V}}$ has a non-meagre Hamel basis.

Proof. Let $X$ be a Banach space in $\mathbf{V}$ of size $\kappa$. We shall show that the forcing extension by $\mathbb{B}_{\lambda}$, where $\lambda=\operatorname{cf}(\kappa)$, adds a non-meagre Hamel basis to $X^{\mathbf{V}}$, even though it does not add bounded subsets to $\lambda$. Note that by Theorem 2.7 we have $\operatorname{cf}(\kappa)>\omega$, hence $\mathbb{B}_{\lambda}$ does not add new reals to the ground model. Also, note that for any $\theta<\lambda$ we have $\theta^{\omega}<\kappa$ by our assumptions, so no subset of $X$ of cardinality $<\lambda$ is dense in $X$. Let us define

$$
H:=\bigcup G, \quad \text { where } G \text { is the generic of } \mathbb{B}_{\lambda} .
$$

Since $G$ is a filter it follows that $H$ is a set of linearly independent vectors. Further, for any $x \in X$, the set $D_{x}=\left\{p \in B_{\lambda}: x \in\langle p\rangle\right\}$ is dense in $\mathbb{B}_{\lambda}$. This implies that $H$ is actually a Hamel basis of $X^{\mathbf{V}}$ in the extension.

Let us suppose for contradiction that $H$ is meagre in the extension. Thus, there exists a condition $q$, a name $\underset{\sim}{C}$ for a dense $G_{\delta}$ set and names ${\underset{\sim}{n}}_{n}$ for dense open sets such that

$$
q \Vdash \underset{\sim}{C}=\bigcap_{n<\omega}{\underset{\sim}{O}}_{n} \text { is dense } G_{\delta} \text { and } \underset{\sim}{H} \cap \underset{\sim}{C}=\emptyset .
$$

So, there exist $\underset{\sim}{x}$ and a rational $\underset{\sim}{r}$ such that $q \Vdash B_{x, r} \subseteq Q_{0}$. Since the cardinality of $q$ is less than $\lambda$ we can find $q_{0} \geq q$ and $x_{0}, r_{0}$ such that

$$
q_{0} \Vdash \bar{B}_{x_{0}, r_{0}} \subseteq B_{x, r} \text { and } \bar{B}_{x_{0}, r_{0}} \cap\langle q\rangle=\emptyset,
$$

and by induction we find $q_{n}, x_{n}$, and $r_{n}$ (for $n<\omega$ ) such that $q_{n+1} \geq q_{n}$ and

$$
q_{n+1} \Vdash \bar{B}_{x_{n}, r_{n}} \subseteq B_{x_{n-1}, r_{n-1}} \cap Q_{n} \text { and } \bar{B}_{x_{n}, r_{n}} \cap\left\langle q_{n}\right\rangle=\emptyset \text {. }
$$

At the end let $p=\bigcup_{n<\omega} q_{n}$ and let $h \in \bigcap \bar{B}_{x_{n}, r_{n}}$. Hence $h \notin\left\langle\bigcup_{n<\omega} q_{n}\right\rangle=$ $\bigcup_{n<\omega}\left\langle q_{n}\right\rangle$. In particular $p \Vdash h \in \underset{\sim}{C}$, so

$$
p \cup\{h\} \Vdash h \in \underset{\sim}{C} \cap \underset{\sim}{H},
$$

which is a contradiction with $p \cup\{h\} \geq q$. 
3.5. An independence result. So far we have seen that in the non-separable case a Hamel basis can be closed, and that in the separable case a Hamel basis cannot even be analytic, but we did not answer the question how complex a Hamel basis of a separable Banach space might be. For example, can a Hamel basis be a projective set? (Recall that a projective set is a set that one gets after successively applying the projection-operator and the complement-operator to a Borel set.) In the following we shall see that the above question is not decidable within ZFC.

THEOREM 3.12. It is consistent with ZFC that no separable Banach space contains a Hamel basis which is a projective set.

The theorem follows from

Lemma 3.13. Suppose that $X$ and $Y$ are Polish spaces, i.e., complete separable metric spaces without isolated points. Then there exists a Borel homeomorphism $f: X \rightarrow Y$ such that $A \subseteq X$ is meagre if and only if $f[A]$ is meagre.

Proof. Let BOREL $(X)$ and BOREL $(Y)$ denote the sets of Borel sets in $X$ and $Y$ respectively, and let $\mathcal{M}_{X}$ and $\mathcal{M}_{Y}$ denote their respective ideals of meagre sets. Since the algebras BOREL $(X) / \mathcal{M}_{X}$ and BOREL $(Y) / \mathcal{M}_{Y}$ are complete and have both countable atomless dense subalgebras, both algebras are isomorphic to the Cohen algebra. In particular, they are isomorphic via the isomorphism $\Phi: \operatorname{BOREL}(X) / \mathcal{M}_{X} \rightarrow \operatorname{BOREL}(Y) / \mathcal{M}_{Y}$. By $[\operatorname{Ke} 95$, Theorem 15.10], the isomorphism is determined by a Borel homeomorphism $g: Y \rightarrow X$ such that $\Phi([A])=\left[g^{-1}(A)\right]$. So, if $A$ is a meagre Borel set in $X$, then $g^{-1}(A)$ is meagre in $Y$.

Proof of Theorem 3.12. Let $X$ and $Y$ be Polish spaces. Then, by Lemma 3.13, all projective sets have the Baire property in $Y$ if and only if the same happens in $X$. In [Sh84] it is proved that if there is a model for ZFC, then there is also one in which all projective sets of reals have the Baire property. Let $X$ be any separable Banach space in this model. Then all projective sets of the separable Banach space $X$ have the Baire property, hence, by Lemma 3.9 and Corollary 3.6, no projective set is a Hamel basis of $X$.

As we have seen in Section 3.3, no separable Banach space has a Hamel basis that is an analytic set. However, it is a well-known result of Arnold Miller in [Mi89, Theorem 9.26] that in Gödel's constructible universe the separable Banach space $\mathbb{R}$ over $\mathbb{Q}$ has a co-analytic Hamel basis. So although Hamel bases in separable Banach spaces cannot be as simple as being analytic, there still can consistently exist a Hamel basis in such a space that is almost as simple, namely co-analytic. Using Miller's technique one can prove a similar statement for all classical Banach sequence spaces, but since both the exact statement and the proof of this result are rather technical in the 
sense of the set theory involved, we decided not to elaborate on this point here.

\section{On complete minimal systems}

4.1. Complete minimal systems versus Hamel bases. As mentioned above, not every Banach space has a c.m.s., while every Banach space has a Hamel basis. Thus, not every Hamel basis is a c.m.s., in fact, a Hamel basis is never a c.m.s.:

Proposition 4.1. No Hamel basis of a Banach space is a complete minimal system.

Proof. Let $H=\left\{h_{\iota}: \iota<\kappa\right\}$ be a Hamel basis of some Banach space $X$. Consider the vector

$$
x=\sum_{i<\omega} 2^{-i} \frac{h_{i}}{\left\|h_{i}\right\|} .
$$

Since $x \in X$, there are $h_{\iota_{0}}, \ldots, h_{\iota_{n}} \in H$ and scalars $a_{0}, \ldots, a_{n}$ such that $x=\sum_{j=0}^{n} a_{j} h_{\iota_{j}}$. Let $k<\omega$ be such that $h_{k} \notin\left\{h_{\iota_{0}}, \ldots, h_{\iota_{n}}\right\}$. Then $h_{k}$ belongs to the closure of the linear span of $H \backslash\left\{h_{k}\right\}$, and therefore $H$ is not a c.m.s. of $X$.

We have also seen (cf. Proposition 3.2) that every Banach space over a complete field has a dense Hamel basis. To the contrary a c.m.s. can never be dense, in fact we get the following

FACT 4.2. A complete minimal system is always nowhere dense.

Proof. Recall that a c.m.s. must be discrete. Let $S \subseteq X$ be a c.m.s. of $X$. For every $x \in S$, let $B_{x, r_{x}}$ be such that $B_{x, r_{x}} \cap S=\{x\}$. Let $\mathcal{O}$ be a non-empty open set in $X$. If $\mathcal{O} \cap B_{x, r_{x}}=\emptyset$ for all $x \in S$, then clearly $\mathcal{O}$ contains a non-empty open set that misses $S$. Otherwise suppose that $x \in S$ is such that $\mathcal{O} \cap B_{x, r_{x}} \neq \emptyset$. Then the open set $B_{x, r} \backslash\{x\} \cap \mathcal{O}$ is a non-empty open subset of $\mathcal{O}$ that misses $S$.

Since a c.m.s. consists of linearly independent vectors, every c.m.s. can be extended to a Hamel basis of the whole space. However, not every Hamel basis contains a subset which is a c.m.s. (since there are Banach spaces which do not have a c.m.s.).

Thus Hamel bases behave very differently than complete minimal systems.

4.2. On $\Phi$-bases. Let us first characterize $\Phi$-bases as linearly ordered sets. The following result was proved in [KPP88]:

TheOREM 4.3. A complete minimal system $\left\{x_{a}: a \in A\right\}$ of a Banach space $X$ is a $\Phi$-basis of $X$ if and only if there exists a linear ordering " $\prec$ " 
on $A$, which we will call uniform, such that

$$
\sup \left\{\mu\left\{x_{a_{k}}: a_{k} \in A, 0 \leq k \leq n\right\}\right\}<\infty,
$$

where the sup is taken over the set of all finite increasing sequences $a_{0} \prec$ $\ldots \prec a_{n}$ in $A$. In addition the order " $\prec$ " on $A$ can be assumed to satisfy

$$
\sup \left\{\mu\left\{x_{a_{k}}: 0 \leq k \leq n\right\}: a_{0} \prec \cdots \prec a_{n}\right\}=M,
$$

where $M$ is a $\Phi$-basis constant of the $\Phi$-basis $\left\{x_{a}: a \in A\right\}$.

Notice that if a linear ordering on $A$ is uniform (with constant $M$ ), then the inverse ordering is uniform as well (with constant at most $1+M$ ).

As a consequence of Theorem 4.3 we get the following (cf. [KPP88, Corollary 2]):

CoROllary 4.4. If $\left\{x_{n}: n<\omega\right\}$ is a $\Phi$-basis in the space $X$ which is not a Schauder basis for any permutation of the indices, then $X$ is representable as the direct sum of two infinite-dimensional subspaces.

Proof. Let $A=\omega$ be the uniformly ordered set. It is enough to show that $A$ can be decomposed into two disjoint infinite subsets $A=A_{0} \cup A_{1}$ such that $a^{\prime} \prec a^{\prime \prime}$ for all $a^{\prime} \in A_{0}$ and $a^{\prime \prime} \in A_{1}$, for then $X=X_{0} \oplus X_{1}$, where $X_{0}=\left[x_{a}: a \in A_{0}\right]$ and $X_{1}=\left[x_{a}: a \in A_{1}\right]$. Indeed, for any $r \in A$ let $D_{r}:=\{a \in A: a \prec r\}$. If there is an $r \in A$ such that both $D_{r}$ and $A \backslash D_{r}$ are infinite, then we are done. So, assume that for each $r \in A, D_{r}$ is either finite or co-finite. Without loss of generality we may assume that the set $I=\left\{r \in A: D_{r}\right.$ is finite $\}$ is infinite. Since $A$ is linearly ordered, $I$ is linearly ordered as well and, by definition of $I$, the order type of $I$ is $\omega$. If $I=A$, then $\left\{x_{n}: n<\omega\right\}$ would be a Schauder basis of $X$, which contradicts the premiss of the corollary. Further, for any $a \in A \backslash I$ and any $r \in I$, by definition of $I$ we have $r \prec a$. If $A \backslash I$ is finite, then a permutation of the indices would give us a Schauder basis of $X$, which again contradicts the premiss of the corollary; thus, $A \backslash I$ is infinite and we can just put $A_{0}=I$ and $A_{1}=A \backslash I$.

The name " $\Phi$-basis" is just an abbreviation for "finitary basis", but since the main feature of $\Phi$-bases is the linear ordering on the index set given by Theorem 4.3 , we could call $\Phi$-bases also linearly ordered bases.

Let us now present some examples of $\Phi$-bases:

1. ([KPP88]) Let $X$ be the space of left continuous functions, defined on $[0,1]$, which have discontinuities of the first kind only at rational points, with the supremum norm. The characteristic functions $x_{q}(t)$ of segments $[0, q]$ (for $q \in \mathbb{Q} \cap[0,1]$ ) form a (countable) $\Phi$-basis in $X$ which is not a Markushevich basis, where a Markushevich basis is a c.m.s. with the additional property that the dual system is total, i.e., $f_{i}(x)=0$ for all $i$ implies $x=0$. 
The next example is well known in non-separable Banach space theory (see, e.g., [Co61, Example 2]).

2. Let $X$ be a (non-separable) Banach space which is constructed as in Example 1 , but any scalar of $[0,1]$ can be a point of discontinuity. Then the functions $x_{a}(t)$ (for $a \in[0,1]$ ) form a $\Phi$-basis in $X$.

3. (cf. [PP90, §7]) Let $B_{p}(1<p<\infty)$ be the space of Besicovitch almost periodic functions. The trigonometric functions $e^{i \lambda t}$ (for $\lambda \in \mathbb{R}$ ) form a $\Phi$-basis in $B_{p}$, with the natural order generated by the real line. This system forms a Markushevich basis and has good approximation properties. In addition it forms an (uncountable) orthogonal basis in $B_{2}$. Related to this example are Questions 5 and 6 in Section 5 .

4. Let us consider the space $X=C\left[0, \omega_{1}\right]$. The characteristic functions of segments $[0, \alpha]$ form a transfinite (hence, a $\Phi$-) basis of $X$, but $X$ does not have a norming Markushevich basis $([\mathrm{AP} \infty])$. Because every Enflo-Rosenthal basis is norming ([P184]), $X$ has no Enflo-Rosenthal basis. (A definition of transfinite bases can be found in [KPP88] or in [Si81].)

5. The natural unit vectors form a Schauder basis in the well-known James space $J$, but $J$ has no unconditional basis (cf. [LT77, p. 25]). The natural unit vectors form a transfinite (hence, a $\Phi$-) basis in the Long James space $X=$ Long $J$ (see also Question 7 ).

6. We can construct the James type spaces $J(\mathbb{Q})$ and $J(\mathbb{R})$ exactly in the same way as $J$ is constructed from $\mathbb{N}$ or Long $J$ from $\left[0, \omega_{1}\right]$. Obviously, the natural unit vectors form again a $\Phi$-basis in these spaces (but see Question 8).

There are Banach spaces having complete minimal systems which are not linearly ordered, but partially ordered by other sets, for example by trees (see [Ja74] or [Hay99]). So we can introduce the following definition: Let $A$ be a partially ordered set. We say that a complete minimal system $\left\{x_{a}: a \in A\right\}$ forms an $A$-ordered basis in a Banach space $X$ if the projections of $X$ onto $\left[x_{b}: b>a\right]$, along $\left[x_{b}: b \ngtr a\right]$, are uniformly bounded on $A$.

Now we give an answer to the first two questions posed in [KPP88] and discuss the third. The questions are the following:

- Does there exist a $\Phi$-basis in $\ell_{\infty}$ ?

- Does each separable Banach space have a $\Phi$-basis?

- Is the existence of a $\Phi$-basis in a Banach space related to its approximation properties?

TheOREm 4.5. Not every separable Banach space has a $\Phi$-basis.

Proof. In [AKP99] it is shown that there exists a separable Banach space which has neither a Schauder basis nor a decomposition into a direct sum 
of infinite-dimensional closed subspaces. This result in combination with Corollary 4.4 gives the proof of the theorem.

Before answering the first question let us recall some definitions. A sequence of closed subspaces $\left\{X_{n}: n<\omega\right\}$ forms a Schauder decomposition of a Banach space $X$ if $\left[\bigcup_{n<\omega} X_{n}\right]=X$ and the projections $P_{n}: X \rightarrow\left[\bigcup_{m<n} X_{m}\right]$ along $\left[\bigcup_{m<n} X_{m}\right]$ are uniformly bounded, which is equivalent to saying that the projections $I-P_{n}$ are uniformly bounded. Obviously, we can enumerate the Schauder decomposition by $1 \leq n \leq \omega$, moving 0 to $\omega$ and shifting $n+1 \rightarrow n$. A Banach space $X$ is called Grothendieck if weak* and weak convergence of sequences in $X^{*}$ coincide. A Banach space $X$ has the Dunford-Pettis property if every weakly compact operator $T$ of $X$ into any Banach space $Y$ maps weakly convergent sequences into norm convergent sequences.

All spaces $C(K)$ where $K$ is a compact extremely disconnected space (hence also their complemented subspaces) are Grothendieck and have the Dunford-Pettis property. In particular, $\ell_{\infty}$ has these properties (cf. [Si81, p. 497]).

Theorem 4.6. Let $X$ be a Grothendieck space with the Dunford-Pettis property. Then $X$ has no $\Phi$-basis.

Proof. Suppose towards a contradiction that $X$ has a $\Phi$-basis $\left\{x_{a}: a \in A\right\}$ and that $A$ is linearly ordered by " $\prec$ ". Obviously, each infinite subset of $A$ contains either an increasing or decreasing infinite sequence. If $\left\{x_{a}: a \in A\right\}$ is a $\Phi$-basis with respect to the order " $\prec$ ", then it is also a $\Phi$-basis with respect to the opposite order " $\succ$ ". So, without loss of generality let us assume that $\left\{a_{n}: n<\omega\right\}$ is such that $a_{n} \prec a_{n+1}$ for all $n<\omega$. Put $X_{0}=\left[x_{a}: a \preceq a_{0}\right]$, for $n>0$ let $X_{n}=\left[x_{a}: a_{n-1} \prec a \preceq a_{n}\right]$, and let $X_{\omega}=\left[x_{a}: \forall n<\omega\left(a_{n} \prec a\right)\right]$. Obviously, $\left\{X_{n}: n \leq \omega\right\}$ is a Schauder decomposition of $X$. But $X$ has no such decomposition (see [De67] or [Si81, p. 497]).

Now let us discuss the connection of $\Phi$-bases with the approximation property. A Banach space $X$ has the approximation property if for every $\varepsilon>0$ and every compact set $K \subseteq X$ there is a bounded linear finite-dimensional operator $T: X \rightarrow X$ such that

$$
\|T x-x\|<\varepsilon \quad \text { for every } x \in K .
$$

Haskell Rosenthal has proved in [Ro85] that a space with a transfinite basis always has the approximation property.

The first step in Rosenthal's proof is the following

Lemma. Suppose that $X_{1}, X_{2}, \ldots$ is a Schauder decomposition of a Banach space $X$ and that for every $n \in \omega, X_{n}$ has the approximation property. Then $X$ has the approximation property. 
The second step is a remark that in order to prove that a space has the approximation property it is sufficient to consider only separable Banach spaces (and hence, only countable transfinite bases).

Finally, the third step is just transfinite induction.

This leads to the following:

(a) Can we use Rosenthal's proof to show that a space with a $\Phi$-basis has the approximation property? How can we describe linearly ordered countable sets which allow the transfinite induction? For example, the union of an increasing and a decreasing sequences, without "overlapping", is good.

(b) On the other hand, there exists a (separable) Banach space which has the approximation property but which does not have the bounded approximation property (cf. [LT77, p. 42]), hence, does not have a finite-dimensional Schauder decomposition. Is there a $\Phi$-basis in that space?

(c) Let $X$ be a Banach space with a c.m.s. which is tree ordered. Does $X$ have the approximation property?

4.3. On Auerbach bases in $\ell_{\infty}$. As mentioned above, every finite-dimensional Banach space has an Auerbach basis. Further it is well known that every separable Banach space has a Markushevich basis (hence, a c.m.s.), but it is still unknown whether every separable Banach space has an Auerbach basis (see also Question 12). On the other hand, a non-separable Banach space even with a c.m.s. may not have an Auerbach basis (cf. [Go85, Theorem 2]), and there exists an Auerbach basis of $c_{0}$ which is not a Markushevich basis (cf. [Go85, p. 223]). Moreover, every non-separable Banach space $X$ with a separable norming subspace in $X^{*}$ admits an equivalent norm $\|\cdot\|$ such that $(X,\|\cdot\|)$ does not have an Auerbach basis (cf. [GLT93]). Thus Auerbach bases are much stronger than ordinary complete minimal systems.

In the following we always assume that the Auerbach basis is normalized. For a set $A \subseteq \mathbb{R}$, we say that $\left\{x_{i}: i \in I\right\} \subseteq \ell_{\infty}$ is an $A$-Auerbach basis of $\ell_{\infty}$ if $\left\{x_{i}: i \in I\right\}$ is a normalized Auerbach basis of $\ell_{\infty}$ and for all $i \in I$ and all $n<\omega$ we have $x_{i}(n) \in A$.

We can prove the following

Proposition 4.7. For $\varepsilon>0$, the space $\ell_{\infty}$ does not have $a[-1+\varepsilon, 1]$ Auerbach basis.

Proof. Assume towards a contradiction that $\left\{x_{i}: i \in I\right\} \subseteq \ell_{\infty}$ is a $[-1+\varepsilon, 1]$-Auerbach basis of $\ell_{\infty}$, and let $\left\{f_{i}: i \in I\right\}$ be the corresponding biorthogonal functionals. Let $\mathbf{1}=(1,1,1, \ldots)$ and let $I_{1}$ be a countable subset of $I$ such that $\mathbf{1} \in\left[x_{i}: i \in I_{1}\right]$. There is a finite set $I_{0} \subseteq I_{1}$ and a vector $y \in\left\langle x_{i}: i \in I_{0}\right\rangle$ such that $\|(\varepsilon / 2) \mathbf{1}-y\|<\varepsilon / 4$, which implies that for any $j \in I \backslash I_{1}$ we have $\left\|x_{j}-y\right\|<1$. Now, since by definition $f_{j}\left(x_{i}\right)=0$ for 
every $i \in I_{0}$, we get

$$
f_{j}\left(x_{j}-y\right)=f_{j}\left(x_{j}\right)-f_{j}(y)=1-0=1,
$$

which contradicts $\left\|f_{j}\right\|=1$.

5. What we would like to know. While writing this paper we came across some problems we could not solve. We think that some of them are quite interesting and working on them could probably give a better understanding of the geometry of Banach spaces, especially of non-separable Banach spaces.

5.1. Questions on Hamel bases. In Section 3 we have seen that every Banach space $X$ in which $\operatorname{cof}\left(\mathcal{M}_{X}\right)$ is less than or equal to $|X|$ contains a non-meagre Hamel basis. In particular, every separable Banach contains a non-meagre Hamel basis. This leads to the following questions:

Question 1. Does there exist a Banach space in which every Hamel basis is meagre? Or is it at least consistent with ZFC that such a Banach space exists?

A related question is whether there exists a Banach space $X$ such that $\operatorname{cof}\left(\mathcal{M}_{X}\right)>|X|$. Now, if topological spaces $X$ and $Y$ are homeomorphic, then both $|X|=|Y|$ and $\operatorname{cof}\left(\mathcal{M}_{X}\right)=\operatorname{cof}\left(\mathcal{M}_{Y}\right)$. Moreover, it is well known that any two Banach spaces of the same weight are homeomorphic (cf. [To81]), and in particular, any Banach space of weight $\lambda$ is homeomorphic to $\ell_{2}(\lambda)$. Thus, the question above is in fact just a question on the existence or nonexistence of a certain cardinal:

Question 2. Is there a cardinal $\lambda$ such that $\operatorname{cof}\left(\mathcal{M}_{\ell_{2}(\lambda)}\right)>\lambda^{\omega}$ ? (Notice that $\lambda^{\omega}=\left|\ell_{2}(\lambda)\right|$.) Or is it at least consistent with ZFC that such a cardinal exists?

Remember that every Banach space over a complete field contains a Hamel basis which is nowhere dense and one which is dense and meagre. Further we have seen that for all classical Banach spaces it is consistent with ZFC that they contain a non-meagre Hamel basis.

In Proposition 2.2 we have seen that the unit sphere of a real Banach space $X$ is not the union of fewer than $\mathfrak{c}$ Hamel bases of $X$. This suggests the following

Question 3. Let $X$ be a real Banach space. Can the unit sphere of $X$ be the union of fewer than $|X|$ Hamel bases of $X$ ? Or is it at least consistent with ZFC that there is a Banach space in which this happens?

The following question was recently investigated in [Hal $\infty$ ], where it is shown that the question is not decidable within ZFC. 
Question 4. Does every real Banach space of cardinality $\kappa$ admit a family of $2^{\kappa}$ different normalized Hamel bases such that the cardinality of the intersection of any two of them is less than $\kappa$ ?

\subsection{Questions on complete minimal systems}

QUESTION 5. As we have seen, the trigonometric functions $e^{i \lambda t}$ (for $\lambda \in \mathbb{R}$ ) form a $\Phi$-basis in $B_{p}$ (where $1<p<\infty$ ), with the natural order generated by the real line. Does this system also form an Enflo-Rosenthal basis in $B_{p}$ ? In particular, does there exist a linear ordering of $\mathbb{Q}$ such that $e^{i \lambda t}$ (for $\lambda \in \mathbb{Q}$ ) is a basic sequence?

Probably not, and probably it is a purely combinatorial question.

The next question is a well known question by Enflo and Rosenthal (see, e.g., [Si81, Problem 17.1]):

Question 6. Does a non-separable space $L_{1}(\mu)$, where $\mu$ is a finite measure, have an Enflo-Rosenthal basis? Or slightly weaker: Does this space have a $\Phi$-basis?

This question was one of the reasons to introduce and investigate in [KPP88] the notion of $\Phi$-bases.

Question 7. Let $X=$ Long $J$. Do the natural unit vectors form an Enflo-Rosenthal basis of $X$ ? Does $X$ have an Enflo-Rosenthal basis?

QUESTION 8. What can we say about geometric properties of the spaces $J(\mathbb{Q})$ and $J(\mathbb{R})$ ? Are the natural unit vectors Markushevich bases in these spaces?

The main question about Auerbach bases is

Question 9. Does $\ell_{\infty}$ have an Auerbach basis?

Probably easier to answer than Question 9 is

Question 10. Does $\ell_{\infty}$ have a $\{-1,1\}$-Auerbach basis, or at least a $\{-1,0,1\}$-Auerbach basis?

It is known (cf. [Hal03]) that $\ell_{\infty}$ has a quotient which is isomorphic to $\ell_{2}(\mathfrak{c})$ and which has a $\{-1,1\}$-Auerbach basis. However, it seems that one cannot extend this Auerbach system to an Auerbach basis of the whole space.

Related to Question 10 is

Question 11. Does $\ell_{\infty}$ have a $\{0,1\}$-c.m.s. (which is a c.m.s. whose vectors consist of 0 's and 1's), or at least a $\{-1,1\}$-c.m.s. or a $\{-1,0,1\}$ c.m.s.?

Still open is also the following question by Pełczyński: 
Question 12. Does every separable Banach space have an Auerbach basis?

\section{References}

[AKP99] Georgi Alexandrov, Denka Kutzarova and Anatolij Plichko, A separable space with no Schauder decomposition, Proc. Amer. Math. Soc. 127 (1999), 28052806.

$[\mathrm{AP} \infty] \quad$ Georgi Alexandrov and Anatolij Plichko, Connection between strong and norming Markushevich bases in nonseparable Banach spaces, preprint.

[Au29] Herman Auerbach, O polu krzywych wypuktych o średnicach sprzężonych [On the area of convex curves with conjugate diameters] (in Polish), Ph.D. thesis, Univ. of Lwów, Poland (at that time), 1930, lost.

[Ba32] Stefan Banach, Théorie des opérations linéaires, Monografie Matematyczne 1, published by M. Garasiński, Warszawa, 1932.

[BJ95] Tomek Bartoszyński and Haim Judah, Set Theory: on the structure of the real line, A. K. Peters, Wellesley, 1995.

[Bi51] R. H. Bing, Metrization of topological spaces, Canad. J. Math. 3 (1951), 175186.

[Co61] Harry H. Corson, The weak topology of a Banach space, Trans. Amer. Math. Soc. 101 (1961), 1-15.

[DJ73] William J. Davis and William B. Johnson, On the existence of fundamental and total bounded biorthogonal systems in Banach spaces, Studia Math. 45 (1973), 173-179.

[Da47] Malon M. Day, Polygons circumscribed about closed convex curves, Trans. Amer. Math. Soc. 62 (1947), 315-319.

[De67] David W. Dean, Schauder decompositions in $(m)$, Proc. Amer. Math. Soc. 18 (1967), 619-623.

[Ed80] Gerald A. Edgar, A long James space, in: Lecture Notes in Math. 794, Springer, 1980, 31-37.

[EK43] Paul Erdős and Shizuo Kakutani, On non-denumerable graphs, Bull. Amer. Math. Soc. 49 (1943), 457-461.

[Go83] Borys V. Godun, Complete biorthogonal systems in Banach spaces, Funktsional. Anal. i Prilozhen. 17 (1983), no. 1, 1-7 (in Russian); English transl.: Funct. Anal. Appl. 17 (1983), 1-5.

[Go84] -, Quasicomplements and minimal systems in $\ell_{\infty}$, Mat. Zametki 36 (1984), 117-121 (in Russian); English transl.: Math. Notes 36 (1984), 551-553.

[Go85] - A special class of Banach spaces, Mat. Zametki 37 (1985), 391-398 (in Russian); English transl.: Math. Notes 37 (1985), 220-223.

[GK80] Borys V. Godun and Mikhail I. Kadets, Banach spaces without complete minimal systems, Funktsional. Anal. i Prilozhen. 14 (1980), no. 4, 67-68 (in Russian); English transl.: Funct. Anal. Appl. 14 (1980), 301-302.

[GLT93] Borys V. Godun, Bor-Luh Lin and Stanimir L. Troyanski, On Auerbach bases, in: Contemp. Math. 144, Amer. Math. Soc., 1993, 115-118.

[GMP83] Leonid N. Gurvits, Volodymyr K. Maslyuchenko and Mikhail M. Popov, Ordinal characteristics of partially ordered sets, report, Chernivtsi State Univ., 1983, 25 pages (in Russian). 
[Hal01] Lorenz Halbeisen, On the complexity of Hamel bases of infinite-dimensional Banach spaces, Colloq. Math. 89 (2001), 133-134.

[Hal03] - A set-theoretic approach to complete minimal systems in Banach spaces of bounded functions, Mat. Studii 20 (2003), 162-166.

$[\mathrm{Hal} \infty] \quad-$, Families of almost disjoint Hamel bases, preprint.

[HH00] Lorenz Halbeisen and Norbert Hungerbühler, The cardinality of Hamel bases of Banach spaces, East-West J. Math. 2 (2000), 153-159.

[Ham05] Georg Hamel, Eine Basis aller Zahlen und die unstetige Lösung der Funktionalgleichung $f(x+y)=f(x)+f(y)$, Math. Ann. 60 (1905), 459-462.

[Hay99] Richard Haydon, Trees in renorming theory, Proc. London Math. Soc. (3) 78 (1999), 541-584.

[Ja74] Robert C. James, A separable somewhat reflexive Banach space with nonseparable dual, Bull. Amer. Math. Soc. 80 (1974), 738-743.

[Je86] Thomas Jech, Multiple Forcing, Cambridge Tracts Math. 88, Cambridge Univ. Press, Cambridge, 1986.

[Je03] —, Set Theory, Springer Monogr. Math., 3rd millenium ed., rev. and expanded, Springer, Berlin, 2003.

[KPP88] Vladimir M. Kadets, Anatolij M. Plichko and Mikhail M. Popov, On a type of complete minimal system in Banach spaces, Izv. Vyssh. Uchebn. Zaved. Mat. 32 (1988), no. 5, 33-40 (in Russian); English transl.: Soviet Math. (Iz. VUZ) 32 (1988), no. 5, 39-48.

[Ke95] Alexander S. Kechris, Classical Descriptive Set Theory, Grad. Texts in Math. 156, Springer, Berlin, 1995.

[Ku83] Kenneth Kunen, Set Theory, an Introduction to Independence Proofs, NorthHolland, Amsterdam, 1983.

[LT77] Joram Lindenstrauss and Lior Tzafriri, Classical Banach Spaces I: Sequence Spaces, Springer, Berlin, 1977.

[Ma45] George W. Mackey, On infinite-dimensional linear spaces, Trans. Amer. Math. Soc. 57 (1945), 155-207.

[Mi89] Arnold W. Miller, Infinite combinatorics and definability, Ann. Pure Appl. Logic 41 (1989), 179-203.

[P180] Anatolij Plichko, A Banach space without a fundamental biorthogonal system, Dokl. Akad. Nauk SSSR 254 (1980), 798-801 (in Russian); English transl.: Soviet Math. Dokl. 22 (1980), 450-453.

[P184] - On bases and complements in nonseparable Banach spaces, Sibirsk. Mat. Zh. 25 (1984), no. 4, 155-162 (in Russian); English transl.: Siber. Math. J. 25 (1984), 636-641.

[PP90] Anatolij Plichko and Mikhail Popov, Symmetric function spaces on atomless probability spaces, Dissertationes Math. 104 (1990).

[Ro85] Haskell P. Rosenthal, Contractively complemented subspaces of Banach spaces with reverse monotone (transfinite) bases, in: Texas Functional Analysis Seminar 1984-1985 (Longhorn Notes), Univ. of Texas Press, Austin, TX, 1985, $1-14$.

[Sh84] Saharon Shelah, Can you take Solovay's inaccessible away?, Israel J. Math. 48 (1984), 1-47.

[Si81] Ivan Singer, Bases in Banach Spaces II, Springer, Berlin, 1981.

[Ta47] Angus E. Taylor, A geometric theorem and its application to biorthogonal systems, Bull. Amer. Math. Soc. 53 (1947), 614-616. 
[To81] Henryk Toruńczyk, Characterizing Hilbert space topology, Fund. Math. 111 (1981), 247-262.

Division of Mathematical Sciences

National Science Foundation

4201 Wilson Blvd

Arlington, VA 22230, U.S.A.

E-mail: tbartosz@nsf.gov

Theoretische Informatik und Logik

Universität Bern

Neubrückstrasse 10

3012 Bern, Switzerland

E-mail: halbeis@iam.unibe.ch

Institute of Mathematics

Cracow University of Technology

Warszawska 24

31-155 Kraków, Poland

E-mail: aplichko@usk.pk.edu.pl
School of Mathematics University of East Anglia Norwich, NR4 7TJ, UK E-mail: M.Dzamonja@uea.ac.uk

Department of Math. Analysis Faculty of Mathematics and Physics Charles University Sokolovská 83

18675 Praha 8, Czech Republic E-mail: murtin@karlin.mff.cuni.cz

Received November 28, 2003

Revised version January 25, 2005 